B. Ehlers

M. Kunz

G. Gross

\section{Dioden-Laser zur Behandlung der} Besenreiser-Varikosis

\author{
Diode-Laser in the Treatment of Spider Veins
}

\section{Zusammenfassung}

Eine Therapie der Besenreiser-Varikosis ist prinzipiell als kosmetische Indikation nach vorherigem Ausschluss oder Therapie einer venösen Insuffizienz indiziert. Mittel der 1 . Wahl ist nach wie vor die Sklerosierungs-Therapie. Falls jedoch die Gefäßlumina für eine sichere Punktion zu klein sind oder die Patienten sich vor Injektionen fürchten, eine Unverträglichkeit des Sklerosierungsmittels besteht oder eine Sklerosierung bereits ohne Erfolg blieb, kann eine Laserbehandlung durchgeführt werden. Neben z.B. dem langgepulsten Farbstoff-Laser und dem frequenzverdoppelten Nd:YAG-Laser (KTP-Laser), die bei der Behandlung von Besenreisern eingesetzt wurden, führt auch der Dioden-Laser nach ca. 2-5 Behandlungen zu einer Koagulation von Gefäßen bis zu $1 \mathrm{~mm}$ Durchmesser. Vorteile gegenüber den zuerst genannten Lasern sind neben der größeren Eindringtiefe auch die geringe Größe und die Wirtschaftlichkeit des Gerätes.

\section{Abstract}

A cosmetic therapy of spider veins is indicated after prior exclusion or therapy of a venous insufficiency. The treatment of first choice is still the sclerotherapy. A laser-treatment can be performed if the vessels are too small for an exact puncture, the patients are afraid of injections, a sclerotherapy was not successful or an intolerance of the ingredients exists. Spider veins can be treated with the long pulsed dye-laser and the frequency-doubled Nd:YAG-laser (KTP-laser). The diode-laser also coagulates vessels up to a diameter of $1 \mathrm{~mm}$ after 2-5 treatments. Advantages of the diode-laser are the greater coagulation depth, the small size and efficiency of the device.

\section{Einleitung}

Besenreiservarizen sind definiert als ständig dilatierte Gefäße, vor allem aus dem subpapillären intradermalen Venenplexus [1]. Innerhalb des Koriums sind sie vom Stratum papillare bis nahe an die Subkutis verteilt. Es handelt sich hauptsächlich um parallel zur Oberfläche verlaufende Gefäße, aber auch um absteigende venöse Arkaden (Abb.1).

Rote Besenreiser liegen in einer durchschnittlichen Tiefe von $0,43 \mathrm{~mm}$ (maximal 0,562 $\mathrm{mm}$ ), blaue Besenreiser in einer durchschnittlichen Tiefe von 0,6 mm (maximal 1,09 mm) [2].
Die pathogenetischen Hauptfaktoren stellen eine lokale Hypertension oder eine konstitutionelle Gefäßwandschwäche dar. Primär können die meist roten kleinlumigen Besenreiser, oder auch Teleangiektasien genannt, unter anderem als vaskuläre Naevi oder essenziell im Laufe des Lebens auftreten. Etwa 90\% der Patienten weisen eine positive Familienanamnese auf [3]. Sie betreffen vorrangig Frauen mittleren Alters. Sekundär können Besenreiser im Rahmen von venösen Insuffizienzen, posttraumatisch, nach Bestrahlung, durch hormonelle Einflüsse oder im Rahmen von internistischen und dermatologischen Grunderkrankungen wie Rosacea, Lupus erythematodes oder Basalzellkarzinom auftreten. Für die Entwicklung von sichtbaren Teleangiektasien spielt die Atrophie der Epidermis mit assoziiertem Verlust der papillären Kapillaren und der Dilatation des subpapillären Plexus eine große Rolle [4]. 


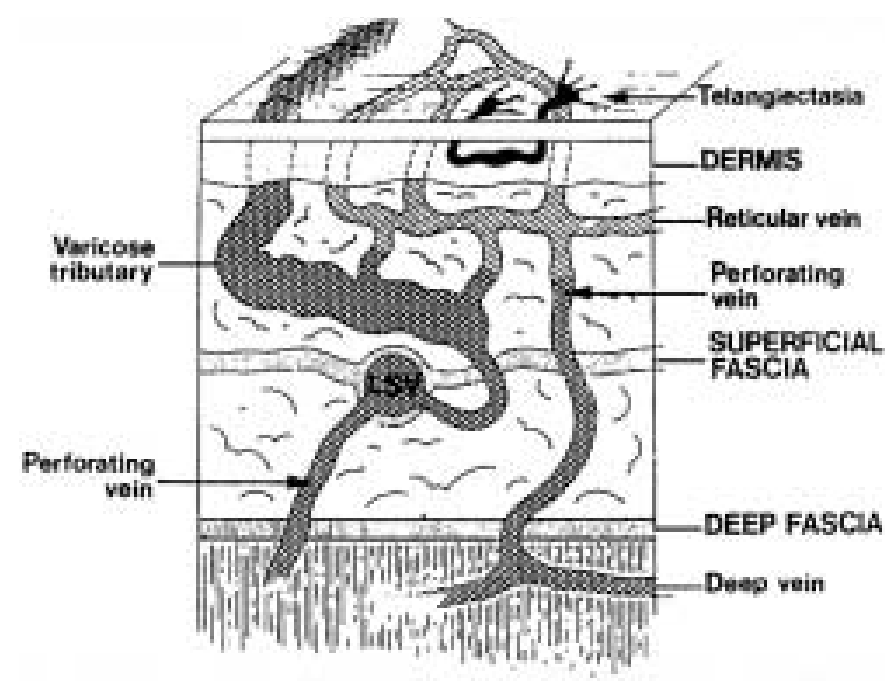

Abb. 1 Schematische Darstellung der Venen in der Haut [26].

Klinisch können rote und blaue Besenreiser unterschieden werden [5].

Rote Besenreiser werden bevorzugt bei jungen Frauen beobachtet. Sie weisen eine höhere Oxygenierung des Hämoglobins auf als blaue Besenreiser und haben einen Gefäßdurchmesser bis zu $1 \mathrm{~mm}$. Sie treten vorrangig als essenzielle Teleangiektasien bei konstitutioneller Gefäßwandschwäche mit Dilatationen der arteriellen Schleife der Kapillaren oder bei Mikro-av-Shunts auf. Als Nebenwirkung nach einer Sklerosierungstherapie kann ein „teleangiektatisches Matting“ mit sehr kleinem Gefäßdurchmes$\operatorname{ser}(<0,2 \mathrm{~mm})$ vorhanden sein.

Inspektorisch erscheinen sie oft als monomorphe, flächenartig wirkende Rötungen durch fein- und gleichkalibrige Teleangiektasien ohne erkennbare Ursprungsgefäße. Sie treten auch einzelstehend auf. Meist verlaufen sie longitudinal mit Querverbindungen.

Blaue Besenreiser betreffen die venösen Schenkel der Kapillaren, weisen eine geringere Flussgeschwindigkeit als die roten Besenreiser auf mit einem Querschnitt von bis zu $2 \mathrm{~mm}$.

Bei überwiegend hormonellen bzw. konstitutionellen Ursachen erscheinen die Besenreiser eher monomorph mit einfacher linearer oder paralleler Anordnung.

Bei der venösen Insuffizienz sind sie durch kleine insuffiziente Vv. perforantes bedingt und treten oft herdförmig als dermale Varizen auf. Sie verlaufen polymorph, fächer-, stern- oder ringförmig, gelegentlich gestreckt mit deutlicher Schlängelung und mit Kaliberschwankungen.

Gestaffelt nach den unterschiedlichen Gefäßdurchmessern wurde eine Klassifikation der Varizen aufgestellt:

\section{Klassifikation nach Duffy (1988) [6]}

Typ I Teleangiektasien, rote Besenreiser 0,1-1 mm $\varnothing$

Typ Ia „Matting“ < 0,2 mm $\varnothing$

Typ II Venektasien, blaue Besenreiser 1-2 mm $\varnothing$
Typ III retikuläre Varikosis 2-4 $\mathrm{mm} \varnothing$

Typ IV Seitenast-Varikosis 3-8 $\mathrm{mm} \varnothing$

Typ V Stamm-Varikosis $>8 \mathrm{~mm} \varnothing$

Erst nach Therapie oder Ausschluss einer venösen Insuffizienz, soll die Behandlung der Besenreiser erfolgen. Eine Doppleruntersuchung zur Prüfung der Funktion der Venenklappen steht im Vordergrund.

Indikationen für die venöse Doppler-Untersuchung:

1. Patienten mit Varizen $>4 \mathrm{~mm}$ Durchmesser

2. Varizen $>2 \mathrm{~mm}$ mit großer Ausdehnung über die Wade oder den Oberschenkel

3. Varizen mit Ausdehnung in die Leiste oder in die Kniekehle

4. sternförmig angeordnete Teleangiektasien, besonders über den üblichen Perforans-Durchtrittsstellen

5. Patienten mit Anamnese einer tiefen Beinvenenthrombose oder Thrombophlebitis

6. Patienten nach erfolgloser Venenchirurgie oder Sklerotherapie

Bei Nachweis von venösen Insuffizienzen erfolgt meist eine Kombinationsbehandlung. Die größeren Varizen werden operiert, die retikulären Varizen sklerosiert. Nach Abschluss können verbliebene Besenreiser, die ohne hämodynamische Relevanz sind, aus kosmetischen Gründen nachbehandelt werden. Obwohl es Berichte gibt, dass Besenreiser zu zyklusabhängigen Beschwerden wie Brennen und Juckreiz führen können, kann ihnen kein echter Krankheitswert zugeordnet werden.

\section{Therapie der Besenreiser}

Das Mittel der 1. Wahl zur Behandlung der Besenreiser ist die Sklerosierung mit z.B. Aethoxysklerol. Erst bei Therapieversagen oder Unverträglichkeit des Sklerosierungsmittels soll eine Laserbehandlung angewendet werden. Ein weiterer Grund für die Laserbehandlung wäre die Angst von manchen Patienten vor Injektionen. Außerdem sind die roten Besenreiser mit ihren geringen Gefäßquerschnitten oft zu klein, um gut und sicher für eine Sklerosierungsbehandlung punktiert zu werden.

In der Literatur sind verschiedene Laser zur Behandlung der Besenreiser beschrieben.

Gute Erfolge wurden mit dem langgepulsten frequenzverdoppelten Nd:YAG-Laser [8] und dem blitzlampengepumpten gepulsten Farbstoff-Laser mit langen Impulsdauern von 1,5 ms [9] erreicht. Weitere Laser sind der Argon-Laser [10] und neuerdings der langgepulste Alexandrite-Laser [11]. Wie bereits eine andere Arbeitsgruppe [7] haben wir zur Behandlung von roten und blauen Besenreisern den Dioden-Laser Medilas D mit einer Wellenlänge von $940 \mathrm{~nm}$ eingesetzt.

\section{Dioden-Laser}

Dioden-Laser werden seit 1962 hergestellt, für den medizinischen Bereich jedoch erst seit wenigen Jahren eingesetzt. Es handelt sich um Halbleiter-Laser, bei denen durch direkte Umsetzung des elektrischen Stroms Photonen erzeugt werden. Das Hauptemissionsspektrum der Dioden liegt bei $630 \mathrm{~nm}$ bis zu $980 \mathrm{~nm}$. Dioden-Laser mit kürzeren Wellenlängen sind zur Zeit nur im unteren Leistungsbereich erhältlich und werden überwiegend für die photodynamische Therapie eingesetzt. Dioden-Laser mit 
längeren Wellenlängen können inzwischen Leistungen von $>50 \mathrm{~W}$ erreichen. Obwohl diese Dioden-Laser eine nicht so hohe Leistungsdichte wie die Nd:YAG-Festkörperlaser aufweisen, stellen sie bei den Indikationen eine gute Alternative zu Nd:YAGSystemen der unteren Leistungsklasse dar. Wegen des hohen Wirkungsgrades entfallen aufwändige, leistungsstarke Netzgeräte und Kühlsysteme, so dass die Dioden-Laser klein und kostengünstig herzustellen sind.

\section{Material und Methoden}

Wir verwendeten zur Behandlung der Besenreiser den 1998 entwickelten Medilas D Dioden-Laser der Firma Dornier mit einer Wellenlänge von $940 \mathrm{~nm}$ und einer maximalen Leistung von $50 \mathrm{~W}$. Appliziert wurde das Laserlicht über eine Laserfaser mit einem Handstück im Non-Kontakt-Verfahren und einer Spotgröße von $1 \mathrm{~mm}$ ohne Überlappung der Einzelspots. Es wurde zur Vermeidung von thermischen Schäden ein gepulster Modus gewählt mit Impulsdauern von 0,05 bis $0,1 \mathrm{~s}$ je nach Tiefe und Dicke der Gefäße. Die Pausenzeit betrug 0,7 s. Die behandelte Oberfläche wurde mehrfach während oder nach der Behandlung gekühlt. Sehr kleine Gefäße wurden während der Behandlung nicht gekühlt, da sonst die Vasokonstriktion die Gefäße nicht mehr erkennen ließ.

Wir behandelten 15 Patienten mit roten und blauen Besenreisern an den Beinen und eine Patientin mit Teleangiektasien im Thorakalbereich. Aufgrund der geringen Fallzahl, der unterschiedlichen Ätiologie und Morphologie der behandelten vaskulären Veränderungen konnte keine statistische Auswertung erfolgen. Exemplarisch werden die Verläufe von 3 Patienten mit roten und blauen Besenreisern und mit Teleangiektasien im Brustbereich dargestellt (Abb. 2-4).

\section{Nachbehandlung und Nebenwirkungen}

Eine zu starke thermische Schädigung der Epidermis und Dermis kann Narben zur Folge haben. Durch kurze Impulszeiten und Oberflächenkühlung kann dieses Risiko deutlich verringert werden. Häufig treten jedoch Blasen und Krusten auf, die aber in der Regel schnell und komplikationslos abheilen.

Eine Sonnenschutz-Creme mit hohem Lichtschutzfaktor für 6 Wochen schützt die Patienten vor Pigmentierungsstörungen, denn besonders unter (zusätzlicher) UV-Einstrahlung können Hyper- oder Hypopigmentierungen auftreten. Meist sind nach einer Laserbehandlung noch Residuen vorhanden, so dass Folgebehandlungen fast immer notwendig sind. Erst nach kompletter Abheilung der laserbedingten Entzündung sollte die nächste Behandlung frühestens nach 6 Wochen geplant werden.

Rezidive sind vorrangig bei venösen Insuffizienzen möglich.

\section{Diskussion}

In der vorliegenden Arbeit konnten wir an Fallbeispielen zeigen, dass der Dioden-Laser zur Behandlung von Besenreisern mit Erfolg eingesetzt werden kann.

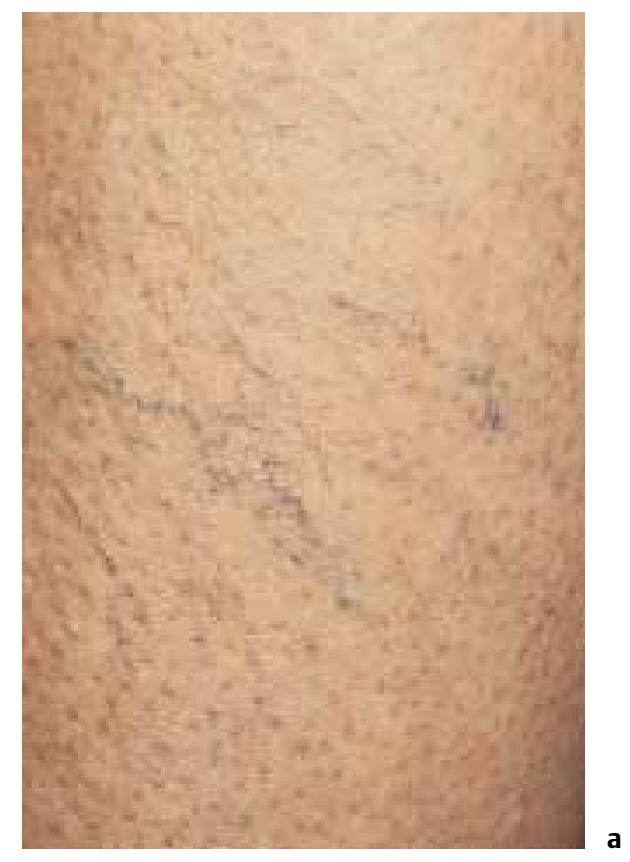

Abb. 2 Patientin 1: Blaue Besenreiser am Unterschenkel. a vor Therapie, b nach 14 Monaten, 6 Dioden-Laserbehandlungen.

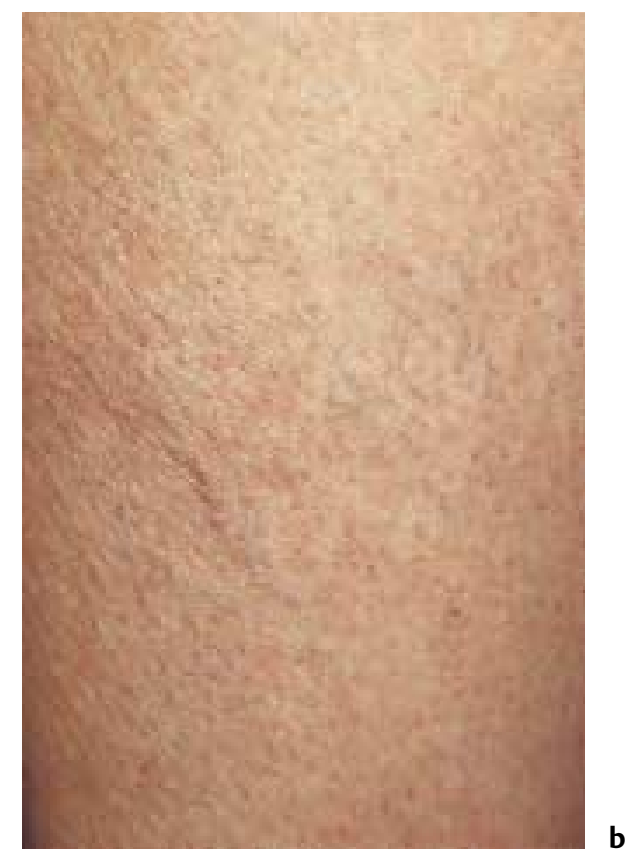

Die klassische Verödung mit Äthoxysklerol gilt für die Behandlung der oberflächlichen Varikosis als Mittel 1. Wahl, wenn eine zuverlässige intravasale Applikation eines Sklerosierungsmittels möglich ist. Meist sind nur 1-2 Behandlungen notwendig. Jedoch treten Komplikationen wie Hyperpigmentierungen bei der Sklerosierung häufiger auf als bei der Laserbehandlung [12] und auch schwerwiegende Nebenwirkungen wie Thrombosierung tiefer Beinvenen, allergische Reaktionen bis hin zum anaphylaktischen Schock und Nekrosen [13] wurden beschrieben. Obwohl eine Laserbehandlung häufig Residuen aufweist und deshalb mehrmals angewendet werden muss, ist sie für Teleangiektasien und dünnlumige blaue Besenreiser die nebenwirkungsärmste Therapiemöglichkeit.

Da die Besenreiser im Bereich der Beine tiefer liegen als im Gesicht, hat der Argon-Laser mit seiner maximalen Eindringtiefe von 1-2 mm [14] und der Gefahr der Narbenbildung und perma- 
Abb. 3 Patientin 2: Rote Besenreiser am Oberschenkel (konstitutionell). a vor Therapie, b nach 5 Monaten, 3 DiodenLaserbehandlungen.
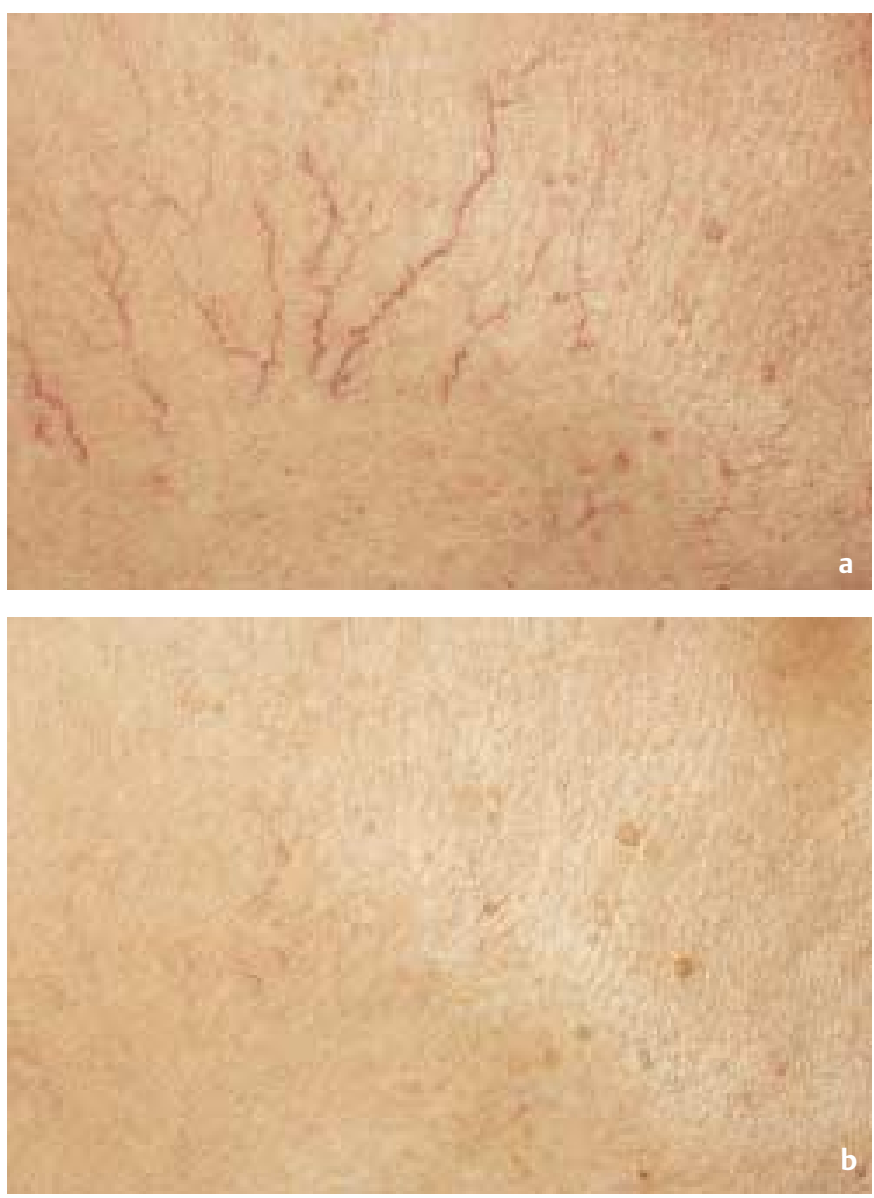

Abb. 4 Patientin 3: Teleangiektasien im Thorakalbereich (konstitutionell). a vor Therapie, b nach 12 Monaten, 3 Dioden-Laserbehandlungen.

Der KTP-Laser (Potassium Titanyl Phosphate) emittiert Licht bei $532 \mathrm{~nm}$. Mit einer Energiedichte von $15-20 \mathrm{~J} / \mathrm{cm}^{2}$ und mit Impulsen im Millisekundenbereich können Teleangiektasien in 1-3 Sitzungen erfolgreich behandelt werden [19-21]. Unerwünschte Wirkungen sind Blasen, purpuriforme Hautveränderungen für ca. 2 Wochen und Pigmentierungsstörungen.

Neuerdings wurden auch über die Anwendbarkeit des langgepulsten Alexandrite-Lasers $(755 \mathrm{~nm})$ bei dieser Indikation berichtet [11] (Energiedichte $20 \mathrm{~J} / \mathrm{cm}^{2}$, Doppelpuls, $1 \mathrm{~Hz}$ ). Nach 3 Behandlungen traten $63 \%$ Gefäßreduktionen auf, besonders der mittleren und kleineren Gefäße.

Neben den beschriebenen Lasern werden auch hochenergetische Blitzlampen mit nicht kohärentem gepulsten Licht zur Besenreisertherapie eingesetzt. Das Photoderm (Wellenlängen 550$590 \mathrm{~nm}$ ) zeigte in einer Studie Erfolge von $75-100 \%$ in $79 \%$ der behandelten Areale [22,23].

Allgemein lässt sich sagen, dass je größer der Gefäßdurchmesser, je livider die Farbe und je tiefer die anatomische Lage der Gefäße ist, desto häufiger muss eine Laserbehandlung durchgeführt werden.

Im Bereich von 600 bis $1300 \mathrm{~nm}$ erfolgt eine nur geringe Absorption von natürlichen Chromophoren und auch eine geringe Lichtstreuung im Gewebe, so dass Laser in diesem Bereich tief ins Gemaximale Eindringtiefe von $0,65 \mathrm{~mm}$ (im Mittel 0,37

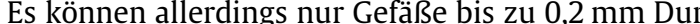
der Fälle ein sehr guter Erfolg ein. Nachteile bestehen durch die postoperative Purpura und mögliche Pigmentierungsstörungen $[17,18]$. 
webe eindringen können, so dass es das „optische Fenster“ für tiefe dermale Läsionen darstellt [24].

Die Eindringtiefe eines Dioden-Lasers bei $805 \mathrm{~nm}$ zeigte ähnliche Eindringtiefen im Gewebe wie der Nd:YAG-Laser (1064 nm) [25]. Da die Absorptionsmaxima von Oxyhämoglobin bei 400, 541 und $577 \mathrm{~nm}$ liegen und für das Melanin unterhalb von $805 \mathrm{~nm}$ liegt, ist zu erwarten, dass die Eindringtiefe bei $940 \mathrm{~nm}$ wie bei dem von uns verwendeten Dioden-Laser dem des Nd:YAG-Lasers vergleichbar ist.

\section{Literatur}

${ }^{1}$ Vanscheidt W, Martay K, Wokalek H, Schöpf E, Leder O. Feinstruktur von Besenreiservarizen. Z Hautkr 1991; 66: 1057-1059

${ }^{2}$ Wiek K, Vanscheidt W, Ishkhanian S, Weyl A, Schöpf E. Selektive Photothermolyse von Besenreiservarizen und Teleangiektasien der unteren Extremität. Hautarzt 1996; 47: 258-263

${ }^{3}$ Boehler-Sommeregger K, Karnel F, Schuller-Petrovic S, Santler R. Do telangiectases communicate with the deep venous system? J Dermatol Surg Oncol 1992; 18: 403-406

${ }^{4}$ Ryan TJ. The epidermis and its blood supply in venous disorders of the leg. Trans St John's Hosp Dermatol Soc 1969; 55: 51 -63

${ }^{5}$ Sommer A, van Mierlo PLH, Neumann HAM, Kessels AGH. Red and blue teleangiectasias. Differences in Oxygenation? Dermato Surg 1997; 23: 55-59

${ }^{6}$ Duffy DM. Small vessel sclerotherapy: an overview. In: Callen et al (eds). Advances in dermatology, vol 3. Chicago: Year Book Medical Publishers, 1988

${ }^{7}$ Kaudewitz P, Klovekorn W, Rother W. Effective treatment of leg vein telangiectasia with a new $940 \mathrm{~nm}$ diode laser. Dermatol Surg 2001; 27 (2): $101-106$

${ }^{8}$ Bethge S, Stadler R. Long-pulsed frequency-doubled neodymium:YAG laser in treatment of superficial varicose veins. Initial clinical experiences. Hautarzt 1999; 50: $181-185$

${ }^{9}$ Hohenleutner U, Wenig M, Walther T, Baumler W, Landthaler M. Treatment of superficial varicosities with a flashlamp-pumped pulsed dye laser with 1,5 ms impulse time. Hautarzt 1998; 49: 560-565
${ }^{10}$ Arndt KA. Argon laser therapy of small cutaneous vascular lesions. Arch Dermatol 1982; 118: 220-224

${ }^{11}$ McDaniel DH, Ash K, Lord J, Newman J, Adrian RM, Zukowski M. Laser therapy of spider leg veins: clinical evaluation of a new long pulsed alexandrite laser. Dermatol Surg 1999; 25: $52-58$

${ }^{12}$ Harnoss BM. Die stadiengerechte Therapie der retikulären und Besenreiser-Varikosis. Angio 1990; 12: 123-126

13 Ostertun H. Risiken der Sklerotherapie. Phlebol Proktol 1988; 17: 140

${ }^{14}$ Spicer MS, Goldberg DJ. Lasers in dermatology. J Am Acad Dermatol 1996; $34: 1-25$

${ }^{15}$ Hohenleutner U, Hilbert M, Wlotzke U, Landthaler M. Epidermal damage and limited coagulation depth with the flashlamp-pumped pulsed dye laser: a histochemical study. J Invest Dermatol 1995; 104: $798-802$

${ }^{16}$ Hohenleutner $U$ et al. Behandlung von Besenreisern mit einem blitzlampengepumpten gepulsten Farbstofflaser mit 1,5 ms Impulsdauer. Hautarzt 1998; 49: 560-565

${ }^{17}$ Bernstein EF et al. Treatment of spider veins with the $595 \mathrm{~nm}$ pulseddye laser. J Am Acad Dermatol 1998; 39: 746 - 750

18 Reichert D. Evaluation of the long-pulsed dye laser for the treatment of leg teleangiectasias. Dermatol Surg 1998; 24: 737-740

19 West TB, Alster TS. Comparison of the long-pulsed dye (590-595 nm) and KTP $(532 \mathrm{~nm})$ lasers in the treatment of facial and leg teleangiektasias. Dermatol Surg 1998; 24: $221-226$

${ }^{20}$ Bethge S, Stadler R. Der langgepulste frequenzverdoppelte Neodymium:YAG-Laser in der Behandlung von Besenreisern. Hautarzt 1999; 50: $181-185$

${ }^{21}$ Adrian RM. Treatment of leg telangiectasias using a long-pulsed frequency-doubled neodymium:YAG laser at $532 \mathrm{~nm}$. Dermatol Surg 1998; 24: 19-23

22 Goldman MP, Eckhouse S. Photothermal sclerosis of leg veins. Dermatol Surg 1996; 22: $323-330$

${ }^{23}$ Hellwig S, Schröter C, Raulin C. Behandlung essentieller Teleangiektasien durch das Photoderm VL. H+G 1996; 71

${ }^{24}$ Anderson RR, Parrish JA. Optical properties of human skin. In: Regan JD, Parrish JA (Eds). The science of Photomedicine. New York: Plenum Press, 1982: 147 - 194

${ }^{25}$ Wyman A et al. Preliminary evaluation of a new high power diode laser. Lasers Surg Med 1992; 12: 506-509

${ }^{26}$ Somjen GM. Anatomy of the superficial venous system. Dermatol Surg 1995; 21 (1): $35-45$ 